\title{
The Effect of Angle of Attack and Flow Conditions on Turbulent Boundary Layer Noise of Small Wind Turbines
}

\author{
Nadiia AFANASIEVA \\ Department of Industrial Safety \\ Faculty of Power Engineering and Communication Systems \\ Don State Technical University (DSTU) \\ Rostov-on-Don, Russia; e-mail: nadiya.afanaseva@yandex.ua
}

(received July 2, 2015; accepted November 3, 2016)

The article aims to solve the problem of noise optimization of small wind turbines. The detailed analysis concentrates on accurate specification and prediction of the turbulent boundary layer noise spectrum of the blade airfoil. The angles of attack prediction for a horizontal axis wind turbine (HAWT) and the estimation based on literature data for a vertical axis one (VAWT), were conducted, and the influence on the noise spectrum was considered. The $1 / 3$-octave sound pressure levels are obtained by semi-empirical model BPM. Resulting contour plots show a fundamental difference in the spectrum of HAWT and VAWT reflecting the two aerodynamic modes of flow that predefine the airfoil self-noise. Comparing the blade elements with a local radius of $0.875 \mathrm{~m}$ in the HAWT and VAWT conditions the predicted sound pressure levels are the $78.5 \mathrm{~dB}$ and $89.8 \mathrm{~dB}$ respectively. In case of the HAWT with predicted local angle of attack ranging from $2.98^{\circ}$ to $4.63^{\circ}$, the acoustic spectrum will vary primarily within broadband frequency band $1.74-20 \mathrm{kHz}$. For the VAWT with the local angle of attack ranging from $4^{\circ}$ to $20^{\circ}$ the acoustic spectrum varies within low and broadband frequency bands $2 \mathrm{~Hz}-20 \mathrm{kHz}$.

Keywords: wind turbine; airfoil self-noise; turbulent boundary layer; flow conditions; angle of attack.

\section{Notations}

$A$ - spectral shape function for TBL-TE noise,

$a-$ radius of tower $[\mathrm{m}]$,

$B$ - spectral shape function for separation (angledependent) noise,

$c$ - chord length $[\mathrm{m}]$,

$c_{0}-$ speed of sound $[\mathrm{m} / \mathrm{s}]$,

$\bar{D}_{h}-$ directivity function for TE noise (high-frequency limit),

$\bar{D}_{l}-$ directivity function for SS noise (low-frequency limit),

$f$ - frequency $[\mathrm{Hz}]$,

$K_{1}, K_{2}, \Delta K_{i}-$ scaled values,

$L$ - span of segment $[\mathrm{m}]$,

$M$ - Mach number, $V_{\mathrm{inf}} / c_{0}$,

$R_{c}$ - Reynolds number based on chord length, $c V_{\text {inf }} / v$,

$R$ - blade radius,

$r_{T}$ - local radius of each blade element [m],

$r_{t}^{\psi}$ - distance from the center of tower to the local blade element in azimuthal position $\psi[\mathrm{m}]$,

$r_{e}$ - slant distance between source and observer [m],

$S_{1}, S_{2}$ - Strouhal numbers defined for TBL-TE and separation noise scaling,

$S t_{p}-$ pressure side Strouhal numbers, $f \cdot \delta_{p}^{*} / V_{\text {inf }}$,
$S t_{s}$ - suction side Strouhal numbers, $f \cdot \delta_{s}^{*} / V_{\text {inf }}$

$V_{0}^{h}$ - oncoming wind speed, at the wind turbine hub height $h[\mathrm{~m} / \mathrm{s}]$,

$V_{c}-$ convection velocity $[\mathrm{m} / \mathrm{s}]$,

$V_{\text {inf }}$ - local induced velocity $[\mathrm{m} / \mathrm{s}]$,

$V(\psi)$ - wind speed as a stream function for tower shadow $[\mathrm{m} / \mathrm{s}]$,

$V_{r}, V_{s}-$ radial and tangential velocity components $[\mathrm{m} / \mathrm{s}]$,

$\alpha_{A}-$ aerodynamic angle of attack [deg],

$\alpha_{\text {stall }}$ - stall angle of attack [deg],

$\beta$ - inflow angle [deg],

$\delta_{p}^{*}$ - pressure side boundary layer displacement thickness $[\mathrm{m}]$,

$\delta_{s}^{*}$ - suction side boundary layer displacement thickness $[\mathrm{m}]$,

$\theta$ - angle from the upstream direction to the blade segment [deg],

$v$ - kinematic viscosity of medium $\left[\mathrm{m}^{2} / \mathrm{s}\right]$,

$\varphi$ - local pitch angle of the blade [deg],

$\psi$ - blade azimuth angle from the upwards vertical [deg],

$\omega-$ angular frequency $[\mathrm{rad} / \mathrm{s}]$.

\section{Introduction}

Developments in field of renewables are needed and wind turbines are important renewable energy systems. 
It can provide a source of clean and renewable electricity for large or small communities. Wind turbine noise is a key factor in determining of the wind energy acceptability. Wind farm developers are facing with a problem of accurate calculation of the noise levels that will arise from the operation of a proposed wind farm development.

At the first stage of calculation the environmentally radiated noise from wind turbines, the noise sources and their levels must be specified. The article concentrates on accurate specification and prediction of the wind turbine turbulent boundary layer (TBL) aerodynamic noise. There is a variety of prediction schemes and approaches for wind turbines TBL noise. LowSON (1992) proposed a classification for the prediction methods.

Class I models give simple estimate of the overall sound pressure level as a simple algebraic function of basic wind turbine parameters.

Class II models are founded on a separate consideration of the various noise mechanisms.

Class III models utilize refined models describing the noise mechanisms and relate them with a detailed description of the rotor geometry and aerodynamics.

The used model should be decided according to the parameter inputs available for the turbine model and also according to the accuracy level needed for the study. The accuracy level needed for the study of the TBL noise is provided sufficiently by Class II models. Class II models are most frequently used and represent the state-of-the-art. Most of these models are based on the work performed by Grosveld (1985), BROOKS et al. (1989) and GLEGG et al. (1987). Using measured surface pressures, Brooks, HodGson (1981) demonstrated that if sufficient information is known about the TBL convecting surface pressure field passing the trailing edge (TE), then TBL noise can be accurately predicted. Schlinker, Amiet (1981) employed empirical description of surface pressure to predict measured noise. LiU, Shamroth (1985) supported a research effort to model the turbulence within boundary layers as a sum of discrete vortex elements. In followup, the authors (BROOKS, HodGson, 1981) matched, measured and calculated mean boundary layer characteristics to prescribed distributions of the discrete vortex elements.

Significant results in modelling of TBL noise were achieved by measuring acoustic properties on wind tunnels. Surface pressure measurements done by Brooks et al. (1989) preceded the most important results as the main contribution to semi-empirical aeroacoustics. In the series of aerodynamic and acoustic measurements on NACA0012 airfoils, they managed to distinguish different sources of TBL noise. The scaling laws based on the results from FFOWCS-WILLIAMS et al. (1970) and BLAKE (1986) formulations were di- vided into two parts: the absolute value, expressed in sound pressure level, and the spectral response. The main feature of this approach is the dependence on boundary layer properties.

Being a pure semi-empirical the so called BPM model (standing for Brooks, Pope and Marcolini) has been used as a benchmark case for the further applications.

Parallel to modelling research effort, there has been a lot of wind turbine testing (in field conditions). OERLEMANS et al. $(2007 ; 2009)$ performed some field tests that pointed out that the main source of noise for a ground observer comes from the down stroke blade. These results also confirmed the influence of outer sections where inflow velocities are higher, in comparison to inner sections.

All these tests revealed trailing edge noise as a main source. The BPM model has become the most significant semi-empirical result in wind turbine aeroacoustics, used as a reference model for new Class III developments and established the basis for airfoil optimization.

\section{Turbulent boundary layer noise prediction methodology}

There is a significant difference in the rotor geometry and aerodynamics of Horizontal Axis (HA) and Vertical Axis Wind Turbines (VAWT) that results in a significant difference in TBL noise arising from the operation of wind turbine. Angle of attack is a fundamental parameter influencing on the flow conditions and the TBL aerodynamic and acoustic properties of rotor blade.

\subsection{Horizontal Axis Wind Turbines}

In the case of conventional, propeller-type HAWT the distribution of angles of attack along the blade and azimuth of rotation can be represented by two basic forms, namely concentric and azimuthal respectively. The concentric distribution reflects a fundamental impact of local geometrical parameters varying along the blade. The angle of attack distribution varying with blade azimuth angle (i.e. the blade rotational angle in the rotor plane) reflects the changes induced by external factors.

Angle of attack defines the point of vortex shedding. The rotating blade is simulated as an airfoil in each cross-section perpendicular to the span. The sketch of velocity vectors appearing on the airfoil is shown in Fig. 1a.

As shown, the inflow angle, $\beta$, is the sum of local pitch angle of blade element, $\varphi$, and the angle of attack, $\alpha_{A}$. The local pitch angle is determined by the blade geometry. The angle of attack is a function of the local inflow velocity $V_{\text {inf }}$, which is result from the 
a)

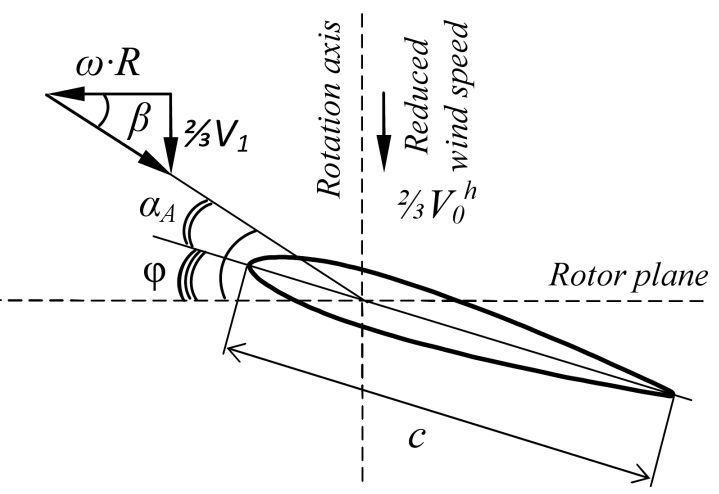

b)

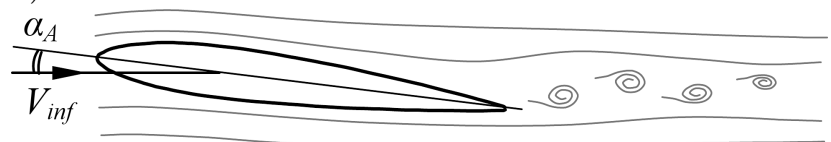

c)

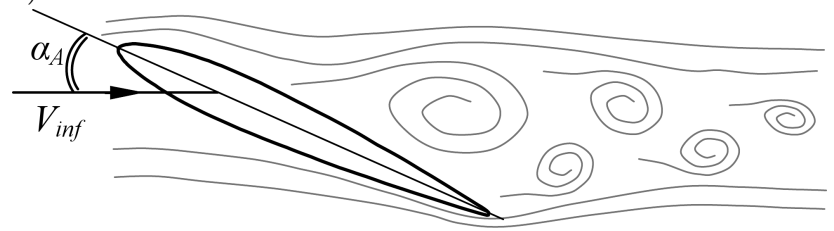

Fig. 1. Schematic of the velocity vectors appearing on airfoil and the flow conditions producing the airfoil selfnoise: a) the velocity vectors appearing on airfoil; b) sketch of turbulent boundary layer trailing edge noise, TBL-TE; c) sketch of turbulent boundary layer separation stall noise, TBL-SS.

oncoming reduced wind speed, $V_{0}^{h} \cdot 2 / 3$, and the rotor speed, $R \cdot \omega$ :

$$
\alpha_{A}=\beta-\varphi=\arctan \left(\frac{2}{3} \cdot \frac{V_{0}^{h}}{R \cdot \omega}\right)-\varphi,
$$

where $\omega$ is the angular velocity of the blade; $V_{0}^{h}-$ oncoming wind speed, at the wind turbine hub height $h$.

One of the main external factors affecting the $\alpha_{A}$ is the WT tower shadow. The tower can be considered as a fixed cylinder in a uniform flow. The stream function, $V(\psi)$, around the cylinder is given by following radial $\left(V_{r}\right)$ and tangential $\left(V_{s}\right)$ components:

$$
\begin{aligned}
& V_{r}=V_{0}^{h} \cdot\left(1-\frac{a^{2}}{r_{t}^{\psi}}\right) \cos (\theta), \\
& V_{s}=-V_{0}^{h} \cdot\left(1+\frac{a^{2}}{r_{t}^{\psi}}\right) \sin (\theta),
\end{aligned}
$$

where $a$ - radius of cylinder (tower cross-section); $r_{t}^{\psi}-$ distance from the center of cylinder (tower) to the local blade element in azimuthal position $\psi$, projected in the tower cross-section plane, $\theta$ - angle from upstream direction $\left(r_{t}^{\psi}, \theta\right.$ are the polar coordinates of the local blade element).
The $r_{t}^{\psi}$ changes with the blade azimuth angle as follows:

$$
r_{t}^{\psi}=R \cdot \sin (\psi),
$$

where $R$ is the blade radius and $\psi$ is the blade azimuth angle from the downwards vertical.

The angle $\theta$ and distance $r_{t}^{\psi}$ can be defined as:

$$
\begin{aligned}
\theta & =\arctan \left(\frac{r_{t}^{180} \cdot \sin (\psi)}{a \cdot(1+n)}\right) \\
r_{t}^{\psi} & =\sqrt{(a \cdot(1+n))^{2}+\left(r_{t}^{180} \cdot \sin (\psi)\right)^{2}} .
\end{aligned}
$$

The distance $r_{t}^{180}$ is fixed at $n$ times the radius of the tower, $a$. The wind speed encountered by the blade, for the stream function given above, can be defined as:

$$
V(\psi)=\sqrt{\left(V_{r}\right)^{2}+\left(V_{s}\right)^{2}} .
$$

Equation (5) only applies for the lower half of the blade cycle, i.e. $0^{\circ} \leq \psi \leq 90^{\circ}$ and $270^{\circ} \leq \psi \leq 360^{\circ}$ from downwards vertical. In practice, the equation will be accurate for angles $0^{\circ} \leq \psi \leq 70^{\circ}$ and $290^{\circ} \leq \psi \leq 360^{\circ}$ (Bullmore et al., 1999).

The variation in wind speed encountered by the blade entails the corresponding variation in angle of attack, $\alpha_{A}$, in accordance with Eq. (1). Thus, the variation in angle of attack depends strongly on the turbine parameters. The $r_{t}^{180}$ distance between the rotor plane and the tower inversely related with differences in angle of attack are recorded as the blade passes the tower.

\subsection{Vertical Axis Wind Turbines}

Compared to conventional HAWT which have to be oriented into the wind direction for the effective operation, the VAWT have some specific advantages. However, VAWT aerodynamic and acoustic properties are not clear. Even in a steady wind stream, flow direction and velocity relative to the rotor blade vary cyclically during each revolution of the rotor (KEIKO et al., 2004).

The angles of attack of VAWT are widely changed during the rotor rotation and are over $20^{\circ}$ even at condition of the effective operation (SouTh, RANGI, 1975; KeIKo et al., 2004; VISBAL, 1990). The experimental results obtained by the KEIKO et al. (2004) for a thin airfoil NACA0018 showed a large separation of the TBL occurred. The flow separation occurred at angle of attack of $9^{\circ}$, large separated flow was observed at angles of attack $12-16^{\circ}$ and strong vortex fields was generated at angle of attack of $20^{\circ}$.

\subsection{Turbulent boundary layer noise prediction model}

Self-noise spectrum of the airfoil both for the case of HAWT and VAWT can be calculated as a function of the angle of attack. 
The impulsive sound waves reflect the initial loading on airfoil in response to an instantaneous change in angle of attack, and include the compression waves on one surface and rarefaction waves on the other.

Boundary layer develops over the airfoil. Noise is produced as the turbulence or vortex shedding passes over the trailing edge. The subsonic flow conditions of concern can be divided into two regimes of flow conditions producing airfoil blade self-noise (BROOKs et al., 1989):

- Flow separation without stalling. For nonzero angles of attack, the flow can separate near the TE on the suction side of the airfoil, producing TBL-TE noise due to the shed turbulent eddies (Fig. 1b).

- Separation stall. At very high angles of attack, the separated flow near the TE gives way to largescale separation, producing TBL Separation Stall (TBL-SS) noise. Wider turbulent boundary layer on the suction side, forming large scale eddies whose interactions with the upper surface of the airfoil introduce pressure fluctuations at lower frequency range (Fig. 1c).

For TBL noise, the noise scales approximately linearly with boundary layer displacement thickness, which is a complex function of angle of attack. Furthermore, the peak frequency of trailing edge noise is inversely related to the displacement thickness, i.e. the peak shifts to lower frequencies as the displacement thickness increases.

Therefore, the relationship between angle of attack and TBL noise is complex, affecting both level and frequency of the noise produced by an airfoil.

Class II models are founded on a separate consideration of noise mechanisms and aim to specify main source of the noise and its level among the various noise mechanisms. In such models, the turbine blade is divided into segments, each segment has its own chord, span, angle of attack, induced velocity, and hence each segment has its own contribution on the total emitted sound level. The number of blade segments is decided according to the changes in the blade geometry along the span.

The Class II model used in article is BPM model that allows study the airfoil self-noise mechanisms as separate sources of noise.

Equation (6) is the main formula for the total TBLTE noise spectrum for the flow separation without stalling in a $1 / 3$-octave presentation.

$$
\begin{aligned}
\mathrm{SPL}_{\mathrm{TBL}-\mathrm{TE}}= & 10 \log _{10}\left(10^{\mathrm{SPL}_{p} / 10}+10^{\mathrm{SPL}_{s} / 10}\right. \\
& \left.+10^{\mathrm{SPL}_{\alpha_{A}} / 10}\right), \quad \text { if } \quad \alpha_{A} \leq \alpha_{\text {stall }},
\end{aligned}
$$

where $\mathrm{SPL}_{p}$ is the sound pressure level of the pressure side, $\mathrm{SPL}_{s}$ is the sound pressure level for the suction side, $\mathrm{SPL}_{\alpha A}$ is the angle-dependent sound pressure level, $\alpha_{\text {stall }}$ is the angle of attack leading to the stall.
The sound pressure level of the TBL noise is directly proportional to the Reynold number. The sound pressure level expressions for pressure side, suction side and separation (angle-dependent) flow are:

$$
\begin{aligned}
\mathrm{SPL}_{p}= & 10 \log _{10}\left(\frac{\delta_{p}^{*} M^{5} L \bar{D}_{h}}{r_{e}^{2}}\right)+A\left(\frac{S t_{p}}{S t_{1}}\right) \\
& +\left(K_{1}-3\right)+\Delta K_{1} \\
\mathrm{SPL}_{s}= & 10 \log _{10}\left(\frac{\delta_{s}^{*} M^{5} L \bar{D}_{h}}{r_{e}^{2}}\right)+A\left(\frac{S t_{s}}{S t_{1}}\right) \\
& +\left(K_{1}-3\right), \\
\mathrm{SPL}_{\alpha_{A}}= & 10 \log _{10}\left(\frac{\delta_{s}^{*} M^{5} L \bar{D}_{h}}{r_{e}^{2}}\right)+B\left(\frac{S t_{s}}{S t_{2}}\right)+K_{2}
\end{aligned}
$$

At angles of attack above $\alpha_{\text {stall }}$ for the separation stall flow conditions the TBL-SS noise spectrum is given by:

$$
\begin{aligned}
\mathrm{SPL}_{\mathrm{TBL}-\mathrm{SS}}= & 10 \log _{10}(\underbrace{10^{\mathrm{SPL}_{p} / 10}}_{=0}+\underbrace{10^{\mathrm{SPL}_{s} / 10}}_{=0} \\
& \left.+10^{\mathrm{SPL}_{\alpha_{A}} / 10}\right), \quad \text { if } \quad \alpha_{A}>\alpha_{\text {stall }}
\end{aligned}
$$

where

$\mathrm{SPL}_{\alpha_{A}}=10 \log _{10}\left(\frac{\delta_{s}^{*} M^{5} L \bar{D}_{l}}{r_{e}^{2}}\right)+A^{\prime}\left(\frac{S t_{s}}{S t_{2}}\right)+K_{2}$,

where $\delta_{p}^{*}$ and $\delta_{s}^{*}$ are the pressure and suction side boundary layer displacement thickness respectively which is function of the Reynolds number and the angle of attack; $M=V_{\mathrm{inf}} / c_{0}$ is the Mach number which varies with local inflow velocity $\left(V_{\text {inf }}\right)$ relative to the speed of sound $\left(c_{0}\right) ; L-$ span of segment, $\bar{D}_{h}$ and $\bar{D}_{l}$ are the directivity functions, where the $h$ or $l$ subscription indicates the high- or low-frequency limit; $r_{e}$ is observer distance.

$A$ and $B$ are spectrum shapes as a function of Strouhal number, $S t$, based on $\delta_{p}^{*}$ and $\delta_{s}^{*}$, which are the responsible factors for obtaining the results in frequency range, $S t_{p}=f \cdot \delta_{p}^{*} / V_{\text {inf }}$ and $S t_{s}=f \cdot \delta_{s}^{*} / V_{\text {inf }}$ are the pressure and suction side Strouhal numbers respectively, $f$ - frequency.

$S t_{2}=S t_{1}\left\{\begin{array}{cc}1 & \left(\alpha_{A}<1.33^{\circ}\right), \\ 10^{0.0054\left(\alpha_{A}-1.33\right)^{2}} & \left(1.33 \leq \alpha_{A} \leq 12.5^{\circ}\right), \\ 4.72 & \left(\alpha_{A}>12.5^{\circ}\right),\end{array}\right.$

$S t_{1}=0.02 M^{-0.6}$.

Finally, $K_{1}, K_{2}$ and $\Delta K_{1}$ are scaled values (BRooKs et al., 1989).

TBL noise mechanism is a source of broadband noise. The characteristic frequency (corresponding to 
the spectrum peak) varies inversely with $\delta^{*}$, that is $f \sim 1 / \delta^{*}$, because the dominant scales of the disturbances (or eddies) within the TBL are of the order of the boundary layer thickness. Pressure fluctuations on the surface should be appropriate primarily to the passage of these eddies. The eddies pass with convection velocity $V_{c}$ give rise to a characteristic frequency $f \sim V_{c} / \delta^{*}$. The convection velocity measured for a given separation distance depends on the eddy scale as compared to the distance traveled. By modelling the field as a distribution of harmonic waves produced by convecting turbulent elements located above the surface (depending on inflow velocity $V_{\text {inf }}$ ) it is assumed that $V_{c} \approx 0.6 \cdot V_{\mathrm{inf}}$ (BROOKS, HoDGsON, 1981).

The velocity dependence of the noise is clearly strong. Thus, the noise source becomes meaningful at the outer elements of a wind turbine blade as OERLEMANS et al. (2007) received in acoustic field measurements on a $2.3 \mathrm{MW}, 94 \mathrm{~m}$ diameter wind turbine.

The flow conditions vary with blade radius. The radius at which the maximum level of the noise occurs is defined as the source radius. Except the highest frequencies, the sources move outward with increasing frequency. It was observed that the source is located at the outer part of the blades, but not at the very tip, approximately at a distance of $1 / 8$ times the radius from the tip. Thus, for instance, the peak location of the blade source moves outward for increasing frequency, from a radius of $21 \mathrm{~m}$ at $315 \mathrm{~Hz}$ to a radius of $26 \mathrm{~m}$ at $5 \mathrm{kHz}$. The observation can be understood using the relation $S t=f \cdot \delta^{*} / V_{\mathrm{inf}} \approx$ const for the trailing edge noise peak; for increasing radius, the local blade inflow velocity $V_{\text {inf }}$ increases and the boundarylayer displacement thickness decreases, so that the produced frequencies are higher (OERLEMANS et al., 2007; Oerlemans, Schepers, 2009). For low frequencies for each source radius the acoustic wavelength is of the same order as the blade chord. Finally, the source distributions show that blade-tower interaction effects are not significant, although at $1.6 \mathrm{kHz}$ a slight noise increase is visible at the location of the tower.

Comprehensive measurements of noise from the Vestas W34, $400 \mathrm{~kW}$ conducted by (Bullmore et al., 1999). Theoretical predictions provide excellent agreement for characteristic frequencies near the peak of the spectrum $1 \mathrm{kHz}$. In the 1 and $2 \mathrm{kHz}$ octave bands modulation is most pronounced. In the $2 \mathrm{kHz}$ octave band modulation is moderately, or well correlated with wind speed. Modulation of noise in the $1 \mathrm{kHz}$ octave band shows a much lower correlation with wind speed. The strength of modulation in both the 1 and $2 \mathrm{kHz}$ octave bands varies strongly with direction. Noise in the $500 \mathrm{~Hz}$ band probably contains both aerodynamic and mechanical components. A tip noise source is present in the intermediate high frequency region between 1.6 and $4 \mathrm{kHz}$.
Within the BPM methodology is determined that the effect of angle of attack is increased primarily in the low frequency range. The spectral peaks tend to shift to lower frequencies with increased angle of attack.

Brooks et al. (1989) have identified spectral peaks by integrating measured spectra. The spectra were truncated at upper and lower frequencies. For the constant-chord airfoil $30.48 \mathrm{sm}$ at $\alpha_{A}=3^{\circ}$ for $V_{\text {inf }}=$ $37.1 \mathrm{~m} / \mathrm{s}$ the $1 / 3$-octave spectrum is peaked between 0.2 and $5 \mathrm{kHz}$. At $V_{\mathrm{inf}}=39.6 \mathrm{~m} / \mathrm{s}$ it is between 0.2 and $10 \mathrm{kHz}$; at $V_{\text {inf }}=55.5 \mathrm{~m} / \mathrm{s}$ the changes in the spectrum are accompanied by an increase in frequency up to the limits $0.5-16 \mathrm{kHz}$, and at $V_{\text {inf }}=71.3 \mathrm{~m} / \mathrm{s}$ the spectrum reaches its peak between 0.8 and $16 \mathrm{kHz}$.

\section{Results and discussion}

Theoretical analysis of published data shows that the flow conditions of the blades are primarily determined by the type of wind turbine performance.

The angles of attack for VAWT are widely changed during the blade rotation and are over $20^{\circ}$ even at condition of the effective operation. This causes the large scale (stall) eddies whose interaction with the upper surface of the airfoil introduce pressure fluctuations at low frequency range. Furthermore, in this case all the segments are moving with equal speed.

In the case of HAWT the TBL spectrum varies along the blade. The term of source radius identifies the segment providing the maximum sound pressure level.

As an example a small HAWT with 2 m rotor diameter, at the nominal rotation speed of $320 \mathrm{~min}^{-1}$ was considered. The yaw error of the turbine was obtained experimentally and equals on average $11.6^{\circ}$ (GURINOV, GAPONOV, 2011). From the above the source radius is located at the outer part of the blade approximately at a distance of $7 / 8$ times the rotor radius. The sours radius can be defined by dividing the blade into the finite number of elements, each one 0.125 times the rotor radius. Thus, the main source is located on 7 th element of the blade with local radius of $0.875 \mathrm{~m}$.

The concentric distribution of the angles of attack which is represented by general Eq. (1) was obtained by the model presented in (AFANASYEVA et al., 2016). The seven segments from the blade root and except the tip, were considered as the following local radius points: $0.17,0.25,0.375,0.5,0.625,0.75$ and $0.875 \mathrm{~m}$.

With using equations (2)-(5) the tower shadow impact for each element was calculated. The resulting plots (Fig. 2) show the predicted angle of attack distribution for a free stream wind speed of $6 \mathrm{~m} / \mathrm{s}$. The difference between the maximum and minimum angles of attack depends on the turbine parameters. The smaller the distance between the rotor plane and tower the greater the differences in angle of attack are recorded 
as the blade passes the tower (Fig. 2a,b). The curve is asymmetric about zero due to the yaw error.

a)

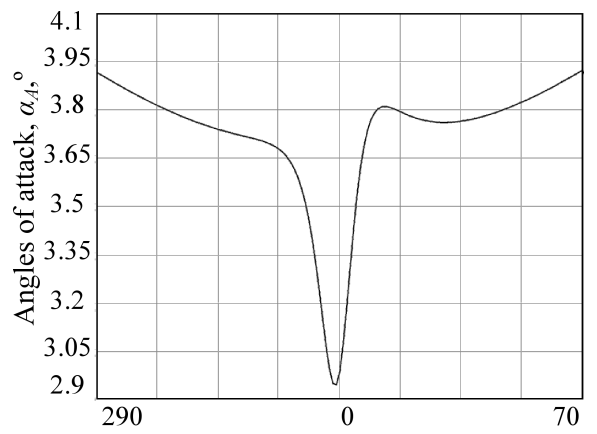

Blade azimuth angle, ${ }^{\circ}$ from downwards vertical

b)

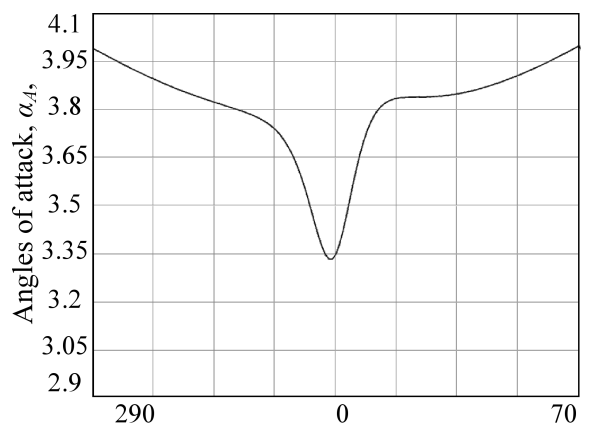

Blade azimuth angle, ${ }^{\circ}$ from downwards vertical

Fig. 2. The tower shadow impact at the main source element (wind speed is $6 \mathrm{~m} / \mathrm{s}$ ), depending on the distance between the rotor plane and tower: two times the tower radius (a), three times the tower radius (b).

The inflow velocities $V_{\text {inf }}$ and the angles of attack $\alpha_{A}$ at the segments will vary along the blade and along the azimuth of rotation. Finally, the angle of attack distribution along the blade span and azimuth of rotation can be imaged by a polar plot (Fig. 3). The azimuthal

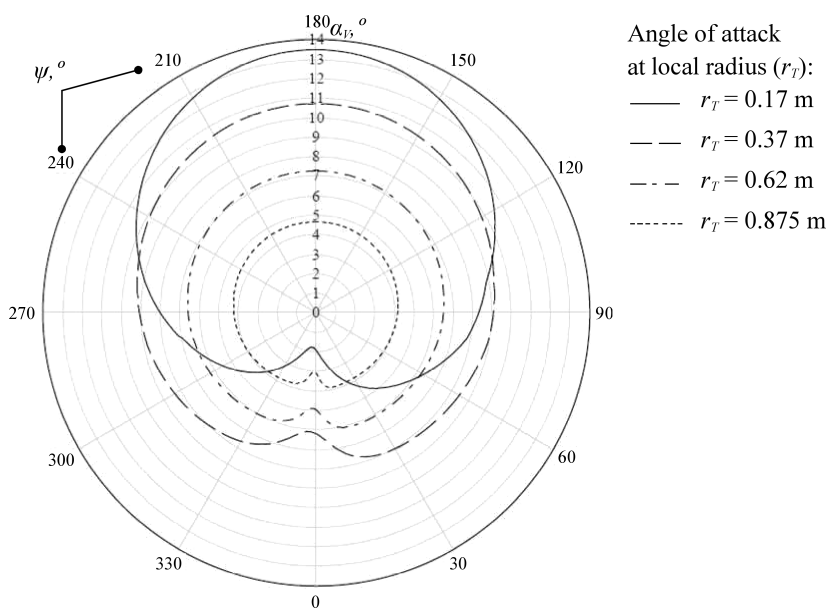

Fig. 3. The angle of attack distribution along the blade azimuth $(\psi)$ at the local radius of each blade element $\left(r_{T}\right)$ (yaw error $-11.6^{\circ}$, rotor diameter $-2 \mathrm{~m}$, wind speed $6 \mathrm{~m} / \mathrm{s}$, the rotor hub height $-5 \mathrm{~m}$, rotor plane is separated from tower to a distance twice the radius of tower $(0.05 \mathrm{~m}))$. distribution of angle of attack has the horizontal asymmetry due to the yaw error of $11.6^{\circ}$ influencing the tower shadow and the vertical asymmetry due to the yaw error, tower shadow and the earth boundary layer.

Thus, the main source of the TBL noise corresponds to the maximum angle of attack $4.63^{\circ}$.

The concentric distribution shows a significant radial-evolution of the angle of attack. With increasing radius the angle of attack decreases. The maximum angle of attack was predicted for the second element and equals $14.3^{\circ}$. The airfoil NACA 0012 starts to stall at about $12.5^{\circ}$. Thus, in case of the HAWT the stall flow mode will appear on the less part of the blade up to the local radius $0.216 \mathrm{~m}\left(\alpha_{A}=12.39\right)$. On the other hand, it can be seen from the previous literature review that the angles of attack of VAWT blades are changed during the rotor rotation from $4^{\circ}$ up to $20^{\circ}$. The large separated flows are observed even at the blade in upstream region (SOUTH, RANGI, 1975; KEIKO et al., 2004; VISBAL, 1990).

It should be noted that there are a lot of external factors that have a significant impact on the angles of attack and their distribution in the rotor plane (Lowson, 1992; Oerlemans et al., 2007; OerleMANS, ScHEPERS, 2009). Thus, a basic prediction allows estimate only the range and trends in distribution of the angles of attack. It seems reasonable to consider the variation of the TBL spectrum for the range of the angles of attack from $0^{\circ}$ to $25^{\circ}$.

BPM model for the TBL noise represented by Eqs. (6)-(12) is applied with the following input parameters: the seven segments are taken with the chord lengths of $0.15,0.138,0.127,0.115,0.103,0.92$ and $0.08 \mathrm{~m}$; span length of each segment equals to 0.125 $\mathrm{m}$; speed of sound $c_{0}=340.29 \mathrm{~m} / \mathrm{s}$; density of air at $20^{\circ} \mathrm{C}, \rho=1.2041$; viscosity of air $v=1.83 \cdot 10^{-5}$. Considered a small wind turbine equipped with blades airfoil NACA 0012.

If we plot the contours of predicted sound pressure levels for a specific local radius depending on the frequency and assuming the angle of attack as varying from $0^{\circ}$ to $25^{\circ}$ then the amplitude-frequency characteristic at the local radius will take the form as shown in Fig. 4.

The resulting contour plots for the TBL-TE noise (the flow separation without stalling) and TBL-SS noise (the separation stall flow conditions) for the local blade radiuses $r_{T}=0.185 \mathrm{~m}$ (Fig. 4a) and $r_{T}=$ $0.875 \mathrm{~m}$ (Fig. 4b) show the predicted sound pressure levels for a broad range of angles of attack.

According to the predicted values of local angles of attack and velocities the $11.6^{\circ}$ yaw error leads to imbalances in azimuthal distribution of the angles of attack. The value of local angle of attack grows in the upward direction within the plane of rotation and varies inversely with the local radius $r_{T}$. So that, the critical values of angle of attack are predicted up to the lo- 
a)

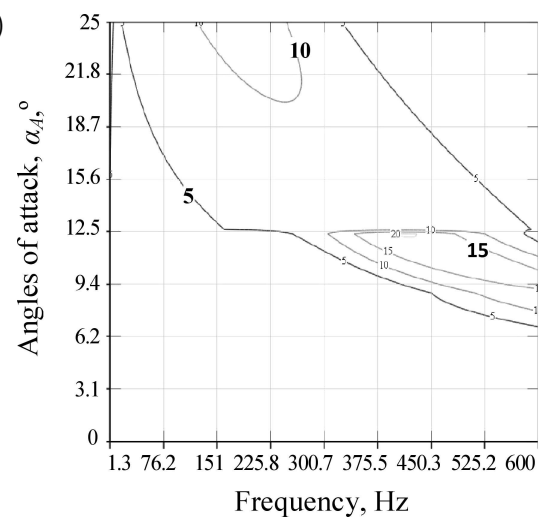

b)

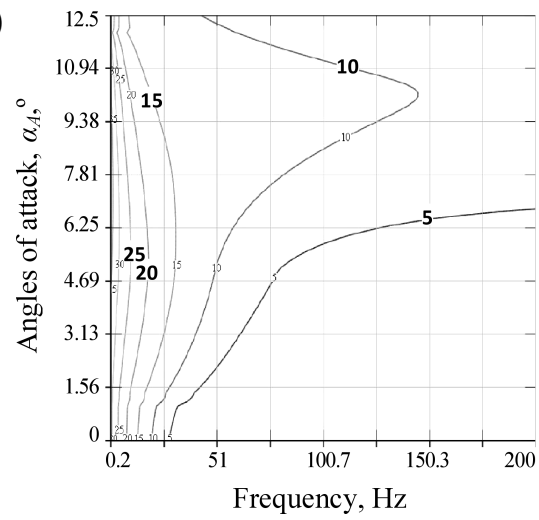

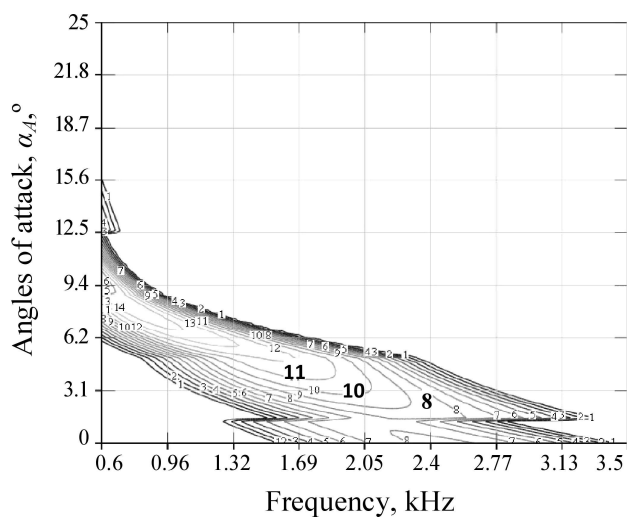

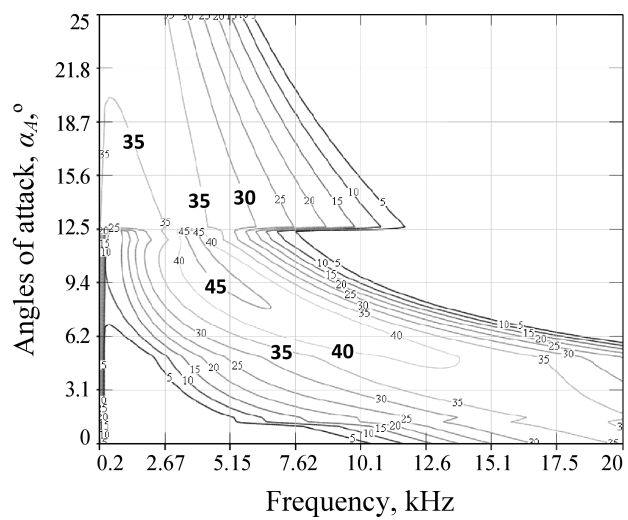

Fig. 4. Resulting contour plots of the TBL-TE (the flow separation without stalling) and TBL-SS (the separation stall flow conditions) noise sound pressure levels $(\mathrm{dB})$ within the low frequency band (left) and the broadband frequency band (right), for each local blade radius, $r_{T}:$ a) $r_{T}=0.185 \mathrm{~m}$; b) $r_{T}=0.875 \mathrm{~m}$.
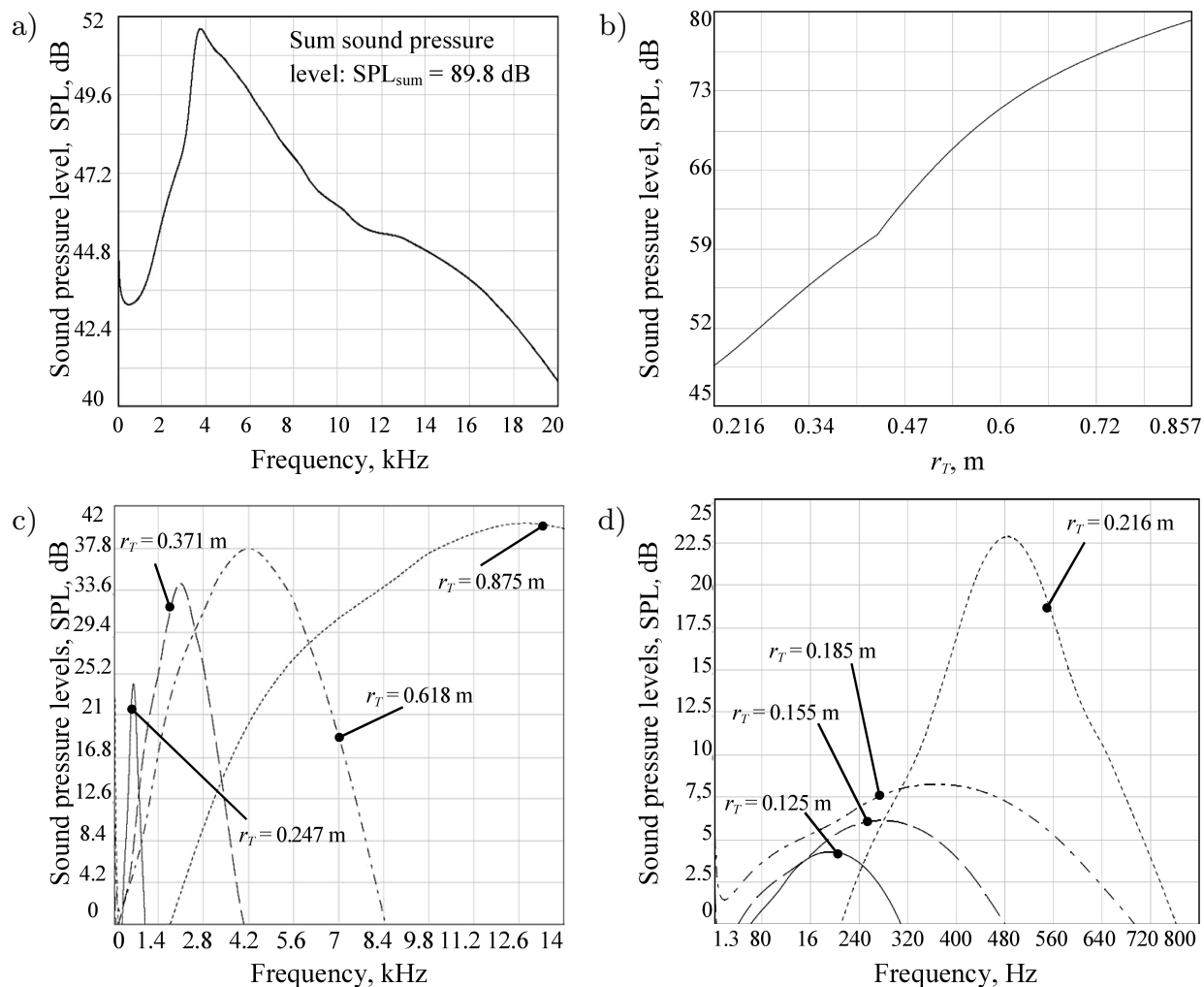

Fig. 5. The TBL sound pressure level, SPL, and the amplitude-frequency characteristic of each local radius, $r_{T}$, depending on the local angle of attack, $\alpha_{A}$ : in the VAWT conditions: a) acoustic spectrum that produced at the local radius $r_{T}=$ $0.875 \mathrm{~m}$; in the HAWT conditions (yaw error (angle), $\alpha_{V}=11.6^{\circ}$; the blade in up position, $\psi=180^{\circ}$; flow separation without stalling): b) the SPL distribution spanwise the blade; c)-d) amplitude-frequency characteristic at each $r_{T}$. 
cal radius $r_{T}=0.216 \mathrm{~m}$. This part of blade will be the source of the TBL-SS noise of flow separation with stall.

Comparing the blade elements with a local radius of $0.875 \mathrm{~m}$ in the HAWT and VAWT conditions the predicted sound pressure levels are $78.5 \mathrm{~dB}$ and $89.8 \mathrm{~dB}$ respectively (Fig. 5a,b).

It is clear that, for example, at local radius $r_{T}=$ $0.185 \mathrm{~m}$ with predicted maximum values of $\alpha_{A}=$ $13.48^{\circ}$ and $V_{\text {inf }}=6.26 \mathrm{~m} / \mathrm{s}$ the acoustic spectrum varies primarily within low frequency band from $16 \mathrm{~Hz}$ to $750 \mathrm{~Hz}$ (Fig. 5d). Finally, all possible frequencies for this local radius within the considered range of angles of attack do not exceed the value of $3.5 \mathrm{kHz}$ (Fig. $4 \mathrm{a}$ ).

On the other hand, in case of the $r_{T}=0.875 \mathrm{~m}$ (Fig. 4b) with predicted maximum values of $\alpha_{A}=$ $4.63^{\circ}$ and $V_{\text {inf }}=27.58 \mathrm{~m} / \mathrm{s}$ the acoustic spectrum will vary primarily within broadband frequency band 1.74$20 \mathrm{kHz}$ (Fig. 5c).

A significant fiche of a VAWT is the fixed rotor radius. Since the angles of attack for VAWT are widely changed during the rotor rotation, large scale separation stall introduce high sound pressure fluctuations at low frequency range. So that, for the VAWT blade element at the $r_{T}=0.875 \mathrm{~m}$ with local angle of attack which varies from $4^{\circ}$ to $20^{\circ}$ the acoustic spectrum will vary within low and broadband frequency bands $2 \mathrm{~Hz}-$ $20 \mathrm{kHz}$ (Fig. 4b).

\section{Conclusions}

The Turbulent Boundary Layer noise calculations were carried out for the small wind turbine equipped by NACA 0012 airfoil. The predicted sound pressure levels for a specific local radius depending on the frequency and the angle of attack were presented in form of contour plots, assuming the angle of attack as varying from $0^{\circ}$ to $25^{\circ}$. The contour plots allow to compare the aeroacoustics characteristics of each local blade radius.

The element of blade defined as the main source of noise for the HAWT was taken at the outer part of the blade (but not at the very tip).

By using the model developed previously the ranges and trends of the angle of attack variation within rotor plane were obtained. Based on these data the $1 / 3$-octave sound pressure levels for the main source element were calculated by semi-empirical prediction model BPM, depending on the flow conditions.

For the conditions of considered HAWT with $2 \mathrm{~m}$ rotor diameter the maximum sound pressure level was found at the local blade radius $r_{T}=0.875 \mathrm{~m}$. The predicted azimuthal distribution of angle of attack allows to estimate the range of its variation during the rotation of the blade. In case of horizontal axis rotor the critical angles of attack appear primarily up to the local radius of $r_{T}=0.216 \mathrm{~m}$. However, due to rela- tively small value of the inflow velocity there are no sound pressure levels of large values at that location. It is clear for this location that the spectrum varies primarily within relatively low frequency band. For example, at local radius of $0.185 \mathrm{~m}$ with predicted value of $\alpha_{A}=13.48^{\circ}$ the noise spectrum ranges from $16 \mathrm{~Hz}$ to $700 \mathrm{~Hz}$.

A significant fiche of a VAWT is the fixed rotor radius.

Comparing the blade elements with a local radius of $0.875 \mathrm{~m}$ in the HAWT and VAWT conditions the predicted sound pressure levels are the $78.5 \mathrm{~dB}$ and $89.8 \mathrm{~dB}$ respectively. In case of the HAWT with predicted maximum local angle of attack $4.63^{\circ}$ and inflow velocity of $27.58 \mathrm{~m} / \mathrm{s}$, the acoustic spectrum will vary primarily within broadband frequency band $1.74-$ $20 \mathrm{kHz}$. For the VAWT with the local angle of attack which varies from $4^{\circ}$ to $20^{\circ}$ the acoustic spectrum will vary within low and broadband frequency bands $2 \mathrm{~Hz}-$ $20 \mathrm{kHz}$.

\section{References}

1. Afanasyeva N.A., Dudnik V.V., Gaponov V.L. (2016), The small horizontal axis wind turbine simulation in yaw conditions, Energy supply and energy efficiency in agriculture. Proceedings of the 10th International Scientific and Technical Conference (21-22 May, 2016, Moscow, VIESH), Moscow, VIESH, 5, 20, $371-376$.

2. Blake W.K. [Ed.] (1986), Mechanics of flow-induced sound and vibration, Frenkiel \& Temple, Inc., Florida.

3. Brooks T.F., Hodgson T.H. (1981), Trailing Edge Noise Prediction From Measured Surface Pressures, Journal of Sound and Vibration, 78, 1, 69-117.

4. Brooks F.T., Pope D.S., Marcolini M.A. (1989), Airfoil Self-Noise and Prediction, NASA Langley Research Center, Hampton, Virginia, NASA RP-1218, 1137.

5. Bullmore A.J., Lowson J.F., Bass J.H., DunBaBIN P. (1999), Wind turbine measurements for noise source identification, Energy Technology Support Unit, Harwell, ETSU W/13/00391/00/REP, 1-346.

6. Ffowcs-Williams J.E., Hall L.H. (1970), Aerodynamic sound generation by turbulent flow in the vicinity of a scattering half plane, Journal of Fluid Mechanics, 40, 4, 657-670.

7. Glegg S.A.L., Baxter S.M., Glendinning A.G. (1987), The Prediction of Broadband Noise from Wind Turbines, Journal of Sound and Vibration, 118, 2, 217239.

8. Grosveld F.W. (1985), Prediction of Broadband Noise from Horizontal Axis Wind Turbines, Journal of Propulsion and Power, 1, 4, 292-299.

9. Gurinov A.S., Gaponov V.L. (2011), Dynamic mathematical model of rotor rotation and the yaw system of a small wind turbine for farms by using the au- 
tomatic orientation system, Vestnik DSTU, T. 11, 10, 61, 1763-1770.

10. Keiko F., Masashi W., Akiyoshi I., Akisato M. (2004), Separation Control of High Angle of Attack Airfoil for Vertical Axis Wind Turbines, Graduate School of Engineering and Department of Mechanical Engineering, Kogakuin University, Japan, 59-62.

11. Liu N.S., Shamroth S.J. (1985), On the Application of a Hairpin Vortex Model of Wall Turbulence to Trailing Edge Noise Prediction, NASA Langley Research Center, Hampton, Virginia, NASA CR-177938, 1-56.

12. Lowson M.V. (1992), Assessment and Prediction of Wind Turbine Noise, Energy Technology Support Unit, Harwell, ETSU W/13/00284/REP, 1-59.

13. Oerlemans S., Sijtsma P., Méndez López B. (2007), Localisation and quantification of noise sources on a wind turbine, Journal of Sound and Vibration, 299, 4-5, 869-883.

14. Oerlemans S., Schepers J.G. (2009), Prediction of wind turbine noise and validation against experiment, International Journal of Aeroacoustics, 8, 555-584.

15. Schlinker R.H., Amiet R.K. (1981), Helicopter Rotor Trailing Edge Noise, NACA Langley Research Center, Hampton, Virginia, NASA CR-3470, 1-145.

16. South P., Rangi R.S. (1975), An Experimental Investigation of a 12-ft Diameter High Speed Vertical-Axis Wind Turbine, National Research Council of Canada, Ontario, Ottawa, Canada, TR-LA-166.

17. Visbal M.R. (1990), Dynamic Stall of a ConstantRate Pitching Airfoil, AIAA Journal of Aircraft, 27, 5, 400-407. 